\title{
Persistence of planetary wave type oscillations in the mid-latitude ionosphere
}

\author{
Jan Laštovička, Petra Šauli and Peter Križan \\ Institute of Atmospheric Physics, Academy of Sciences of Czech Republic, Prague, Czech Republic
}

\begin{abstract}
Planetary wave type oscillations have been observed in the lower and middle atmosphere but also in the ionosphere, including the ionospheric $F 2$ layer. Here we deal with the oscillations in $f o F 2$ analysed for two Japanese and two US stations over a solar cycle (1979-1989) with the use of the Morlet and Paul wavelet transforms. Waves with periods near 5, 10 and 16 days are studied. Only events of duration of three wave-cycles and more are considered. The results are compared with the results of a similar analysis made for $f o F 2$ and the lower ionosphere over Europe (Laštovička et al., 2003a,b). The 5-day period wave events display a typical duration of 4 cycles, while the 10- and 16-day wave events are less persistent with typical duration of about 3.5 cycles and rather 3 cycles, respectively, in all three geographic regions. The persistence pattern in terms of number of cycles and in terms of number of days is different. In terms of number of cycles, the typical persistence of oscillations decreases with increasing period. On the other hand, in terms of number of days the typical persistence evidently increases with increasing period. The spectral distribution of event duration is too broad to allow for a reasonable prediction of event duration. Thus the predictability of the planetary wave type oscillations in foF 2 seems to be very questionable. The longitudinal size of the planetary wave type events increases with increasing wave period. The persistence of the planetary wave type events in foF2 and the lower ionosphere is similar in Europe, but the similarity in occurrence of individual events in $f o F 2$ and the lower ionosphere is rather poor.
\end{abstract}

Key words ionosphere-atmosphere interaction mid-latitude ionosphere - ionospheric disturbances atmospheric waves

\section{Introduction}

Various processes in the lower-lying layers of the atmosphere, particularly in the troposphere, summarized for simplicity under the term "meteorological processes', can affect the ionosphere basically through two channels: i) electrical and electromagnetic phenomena, and ii) upward propagating waves in the neutral atmosphere. We treat the latter category, with upward propagating

Mailing address: Dr. Jan Laštovička, Institute of Atmospheric Physics, Academy of Sciences of Czech Republic, Bocni II, 14131 Prague, Czech Republic; e-mail: jla@ufa.cas.cz waves in the neutral atmosphere, which are more important from the point of view of energy deposition and atmospheric modification than the phenomena under (i). Namely we focus on the effects of planetary waves on the ionosphere.

Various tropospheric and to a limited extent stratospheric and mesospheric «meteorological» processes and periodic solar heating and cooling excite waves in the neutral atmosphere. Upward propagating waves in the neutral atmosphere and their modifications, interactions and modulations affect the ionosphere, when and if they reach it. Those waves are planetary waves, tidal waves, gravity waves, and infrasonic waves. Most of the 'meteorological influences' on the ionosphere arise from the upward propagating gravity, tidal and planetary waves (e.g., Kazimirovsky et al., 2003). The meteorological influences play an important role in the overall ionospheric variability (e.g., Forbes et al., 2000; Rishbeth and Mendillo, 2001). Lašto- 
vička (2006) briefly reviewed effects of atmospheric waves (planetary, tidal, gravity and infrasonic) on the ionosphere.

Planetary waves (periods of about 2-30 days) are very predominantly of tropospheric origin and can penetrate directly to heights slightly above $100 \mathrm{~km}$. They have to propagate upwards into the $F$-region ionosphere via an indirect way. Their effects were observed in the lower ionosphere (e.g., Laštovička et al., 1994), in the ionospheric $E$ region in $h^{\prime} E$ (e.g., Cavalieri, 1976) and sporadic- $E$ layer (e.g., Haldoupis et al., 2004), and in the $F 2$ region (e.g., Forbes and Zhang, 1997; Altadill and Apostolov, 2001, 2003; Laštovička et al., 2003b; Altadill et al., 2004).

Typical planetary wave periods are broad spectral peaks around 2, 5, 10 and 16 (very broad spectral peak) days, but the planetary wave spectrum is very variable and on individual days it can be much different. They roughly correspond to eigenfrequencies of the atmosphere, which slightly differ for various modes; for instance for the wave with the zonal wave number 1 they attain values of 1.2, 5, 8 and 12 days (these periods are Doppler shifted by the prevailing wind). All planetary wave periods are quasi-periods with the exact period varying within a period range. Amplitudes of planetary waves are unstable, as well; planetary waves typically occur in bursts of a couple of waves. The duration of such wave bursts in the critical frequency of the $F 2$ region, $f_{o} F 2$, in other words the persistence of the planetary wave type events, is the main topic of the paper. Laštovička et al. (2003b) made such investigations for four representative European stations listed in table I. The primary aim of this paper is to make such investigations for representative stations from the mid-latitude U.S.A. and Japan in comparison with our previous results on the persistence of planetary wave events over Europe.

It should be mentioned that in the $F$ region it is correct to use the term planetary wave type oscillations, because not all oscillations in the planetary wave period range are related to planetary waves in the neutral atmosphere; some of them are caused by periodic variability of geomagnetic activity (e.g., Altadill and Apostolov, 2003). Planetary waves cannot propagate directly to the F2 region heights. They have to propagate indirectly via modulation of other agents like upward propagating tides or gravity waves, vertical drifts, or the turbopause height and its properties.

Laštovička et al. (2003a) studied the persistence of planetary wave events in the lower ionosphere over Europe. Data and methods used by Laštovička et al. (2003a,b) allow examine the possible similarity of planetary wave type oscillations in the lower ionosphere and $f o F 2$, which is the second topic of the paper. The third topic is to use similarity and/or dissimilarity of planetary wave events in foF 2 over Europe, Japan and the U.S.A. for rough estimate of the longitudinal size of planetary wave type events.

\section{Data and methods}

As the basic ionospheric parameter we use the main characteristics of the $F 2$ region, the

Table I. Co-ordinates of ionospheric stations and missing intervals from period 1979-1989.

\begin{tabular}{cccc}
\hline \hline Station & Geographic latitude & Geographic longitude & Missing intervals \\
\hline Boulder & $40.0^{\circ} \mathrm{N}$ & $105.3^{\circ} \mathrm{W}$ & $1982 / 1983$ \\
Wallops Island & $37.9^{\circ} \mathrm{N}$ & $75.5^{\circ} \mathrm{W}$ & $1979,1987 / 1988,1988,1988 / 89,1989$ \\
Akita & $39.7^{\circ} \mathrm{N}$ & $140.1^{\circ} \mathrm{E}$ & $1984 / 1985,1988 / 1989,1989$ \\
Wakkanai & $45.4^{\circ} \mathrm{N}$ & $141.7^{\circ} \mathrm{E}$ & $1988 / 1989$ \\
Juliusruh & $54.6^{\circ} \mathrm{N}$ & $13.4^{\circ} \mathrm{E}$ & \\
Slough & $51.5^{\circ} \mathrm{N}$ & $0.6^{\circ} \mathrm{W}$ & $1988 / 1989,1989$ \\
Průhonice & $50.0^{\circ} \mathrm{N}$ & $14.6^{\circ} \mathrm{E}$ & 1979 \\
Rome & $41.8^{\circ} \mathrm{N}$ & $12.5^{\circ} \mathrm{E}$ & \\
\hline
\end{tabular}


widely available critical frequency foF 2 . Data from four representative stations in Europe, two in the U.S.A. and two in Japan are used over the period 1979-1989 (from maximum to maximum of the solar cycle). The co-ordinates of those stations are listed in table I. Noontime (10-14 LT) average values of foF2 are used. Sometimes there are problems with quality and availability of $f o F 2$ data with some stations for some periods (e.g., Burešová, 1997). The above stations and the analyzed period were selected with respect to reduction such problems to minimum. Therefore good quality data with minimum gaps are used in the paper. Single data gaps were interpolated with the use of data of the same station at other local times and/or from neighboring stations at the same time, or combination of both, based on availability of data. Nevertheless, some intervals have not been taken into analysis due to more data gaps. The selection of the optimum period was made for European stations, therefore the number of missing data intervals for the US and Japanese stations are larger.

For the «vertical» comparison of the planetary wave type oscillation events in Europe we need data representing the lower ionosphere. The radio wave absorption measurements obtained by the A3 method utilizing the oblique wave propagation are used. Data from two radio paths from central Europe are used. The path Luxembourg-Panská Ves has parameters as follows: $f=6.09 \mathrm{MHz}, f_{\mathrm{eq}}=2.1-2.2 \mathrm{MHz}$ (equivalent vertical frequency), reflection point $50^{\circ} 04^{\prime} \mathrm{N}, 10^{\circ} 18^{\prime} \mathrm{E}$, transmitter-receiver distance $610 \mathrm{~km}$. The path Deutschlandfunk-Panská Ves has different parameters: $f=1539 \mathrm{kHz}, f_{\mathrm{eq}}=650$ $700 \mathrm{kHz}$, reflection point $50^{\circ} 16^{\prime} \mathrm{N}, 11^{\circ} 47^{\prime} \mathrm{E}$, transmitter-receiver distance $390 \mathrm{~km}$. The absorption along the former radio paths is formed very predominantly at altitudes of about 90-100 $\mathrm{km}$, whereas the latter absorption is formed mostly at altitudes of about $85-90 \mathrm{~km}$.

As a mathematical tool for the description of the persistence of planetary wave type oscillations within time series of foF we use wavelet analysis (Torence and Compo, 1998; Vidakovic, 1999). The Continuous Wavelet Transform (CWT) is an excellent tool for analysing changing properties of non-stationary signals. In analyses of signals, the wavelet representations allow us to view a time-domain evolution in terms of scale/period components. The CWT is a convolution of the data sequence with scaled and translated version of the mother wavelet. Varying the wavelet scale and translating along the localised time is possible to construct a picture showing both the amplitude of any feature versus the scale/period and how this amplitude varies with time. For a thorough introduction to wavelet transforms, the reader is referred, e.g., to Mallat (1998).

The wavelet transform of the data $X(t, z)$ is defined as

$$
T_{X}(a, t, z)=\int_{R} X(u, z) \frac{1}{\sqrt{a}} \psi_{0}\left(\frac{u-t}{a}\right) d u \quad a>0, z \in Z
$$

where $\psi_{0}(t)$ is a mother-wavelet. In the present work, we used Morlet and Paul (complex) mother-wavelets, defined as

Morlet: $\psi_{0, N}(t)=\left(\pi \sigma^{2}\right)^{-1 / 4} \exp \left(-\frac{t^{2}}{2 \sigma^{2}}\right) \exp \left(i 2 \pi v_{0} t\right)$
param $=2 \pi \sigma v_{0}$

Paul: $\psi_{0, N}(t)=\frac{2^{N} i^{N} N !}{\pi(2 N) !}(1-i t)^{-(N+1)}$

param $=N$

where param counts the number of oscillations of the (real part of the) wavelet and provides the user with a degree of freedom that can be easily tuned to a given purpose. Wavelets, as building blocs of models, are well localized in both time and scale (period, frequency). As the Paul wavelet decays more quickly in the time-domain then Morlet, it enables better time localization. The greater difference is in frequency/ period domain. For the given count of evident oscillations in the wavelet the Paul wavelet is much less efficient. As a source Matlab code the wavelet software for Morlet wavelet computations provided by Torrence and Compo was used. Matlab codes are available at URL: http://paos.colorado.edu/research/wavelets/.

Since Laštovička et al. (2003a) used only less suitable Meyer wavelet in analysis of the planetary wave activity in the lower ionosphere, we use for the «vertical» comparison over Eu- 
rope only the results of the Mayer wavelet transform as published by Laštovička et al. (2003a,b). The Mayer (e.g., Daubechies, 1994) and Morlet wavelet transforms yielded the same statistical characteristics of the planetary wave type event persistence for foF 2 over $\mathrm{Eu}-$ rope (Laštovička et al., 2003b). Commercial MATLAB-Wavelet software has been used to compute the Meyer wavelet transform.

The wavelet analysis is applied to consecutive 1-year long intervals, shifted by half a year (January-December 1979, July 1979-June 1980, January-December 1980, etc.). Thus for Slough and Juliusruh we have 21 partly overlapping intervals for the period 1979-1989, for other stations a bit less due to data gaps. Only wave events with duration of at least three wave cycles are taken into account.

\section{Persistence of planetary wave type events in $f o F 2$}

Figure 1 presents an example of the normalized wavelet power spectrum for the Morlet wavelet transform. The Morlet wavelet transform results are evaluated for the normalized absolute power, i.e. we search for intervals with values larger than a fixed value. In terms of colours in fig. 1 it means that we consider only intervals with yellow and red colour of the duration of at least three wave cycles. Only results inside of the conus-of-influence (black thin curve) are taken into account. The border effects affect the information out of the thin curve. Even though the values shown in fig. 1 are normalized, the way of their interpretation with respect to a fixed level is hereafter called «ab-
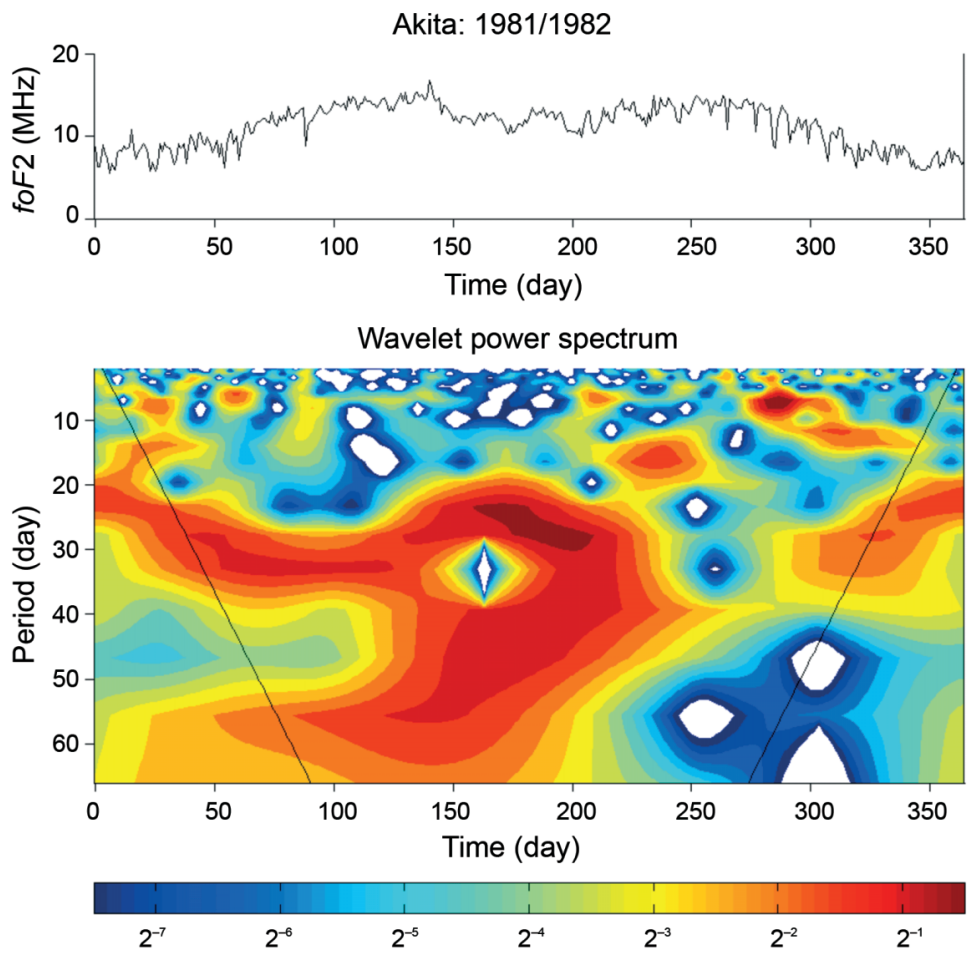

Fig. 1. Planetary wave type activity inferred from $f o F 2$ for Akita, 1 July 1981-30 June 1982, Morlet wavelet transform. Top panel: time series of raw $f o F 2$ data. Bottom panel: wavelet power spectrum of the planetary wave activity changing by colour from white and black-blue (minimum values) through green to red and black-red (maximum values). Wavelet power spectrum is normalized to 1 . 

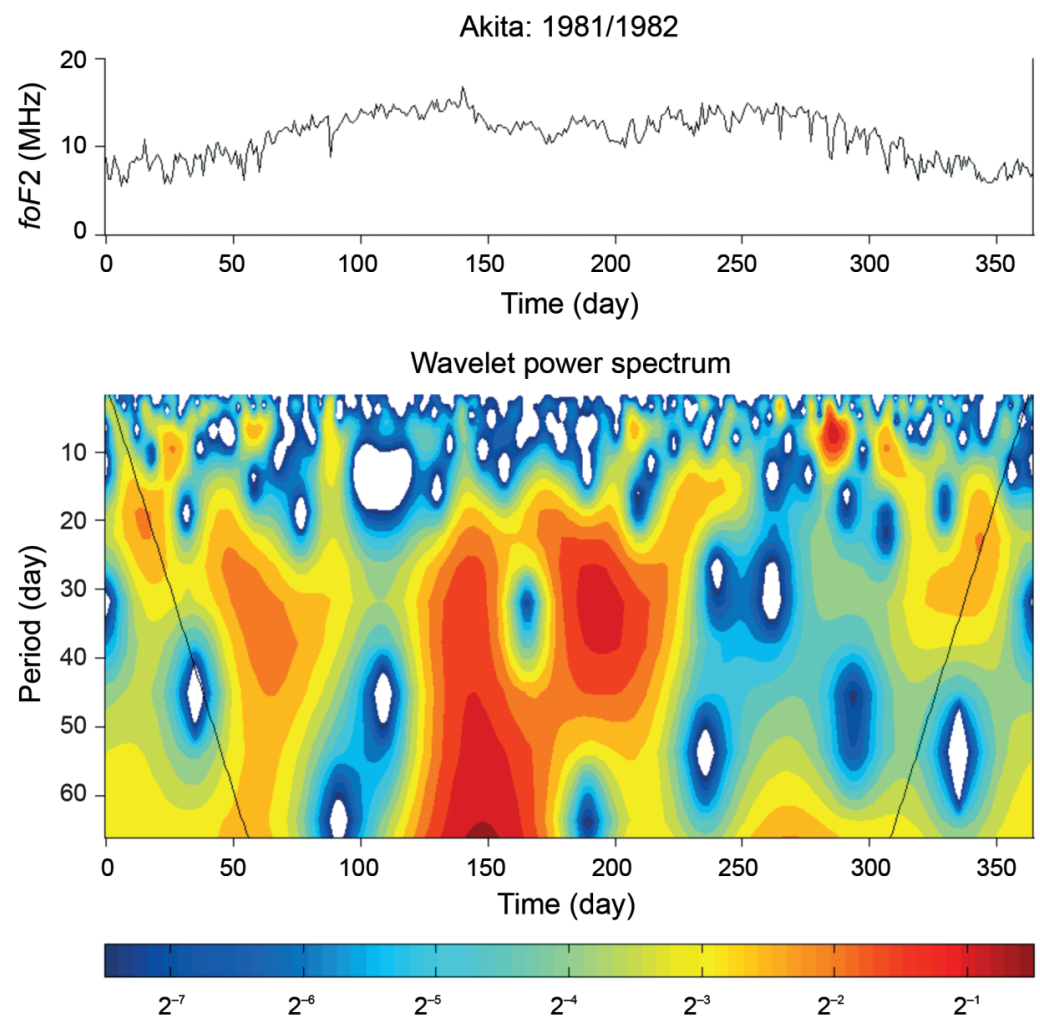

Fig. 2. The same as fig. 1 but for Paul wavelet.

solute» power. However, the Morlet wavelet transform results are also evaluated in terms of «relative» power, when the seasonally variable level of the background foF 2 , shown in the top panel, is taken into account. We consider relative intensifications with respect to the vicinity of the events with duration of at least three wave cycles to be planetary wave type events. This allows us to compare the results of the relative and absolute power approach to interpretation of the results as it was done for European data by Laštovička et al. (2003b). Figure 2 shows an example of the results of the Paul wavelet transform. The results of the Paul wavelet transform are evaluated in terms of the «absolute» power in the same way as Morlet results.

The most pronounced feature of figs. 1 and 2 is a large temporal and partly spectral variability of the planetary wave activity. The migration of periods of planetary wave activity is also well visible. The large temporal variability of planetary wave activity has to result in a limited persistence of the individual planetary wave events.

The statistics of duration of individual events of the enhanced planetary wave activity for all yearly intervals and period bands centred at 5, 10 and 16 days is summarized in tables II, III and IV for Paul transform, «absolute» evaluation, Morlet transform, «absolute» evaluation and Morlet transform, «relative» evaluation, respectively. The tables present the number of events together with their mean, median and the most often occurring number of cycles for individual stations and the average values for all four stations.

Tables II-IV reveal for the 5-day wave the typical persistence of well-developed wave events to be of 4 cycles consistently for all three ways of analysis. For the 10-day wave, both the 
Table II. Statistics of persistence of planetary wave type oscillations in foF 2 for stations from the Northern America and Japan based on the Paul wavelet transform («absolute» evaluation). The average values for medians and the most frequent values are presented with a step of 0.5 .

\begin{tabular}{cccccc}
\hline \hline Station & Period (days) & Number of events & Median value & Mean value & Most frequent value \\
\hline \multirow{2}{*}{ Boulder } & 5 & 24 & 4 & 4.3 & 4 \\
& 10 & 15 & 4 & 4.0 & 3.5 \\
Wallops Island & 16 & 20 & 3.5 & 3.9 & 3 \\
& 5 & 20 & 4 & 4.2 & 4 \\
Wakkanai & 10 & 15 & 3.5 & 3.6 & 3.5 \\
& 16 & 16 & 3.5 & 3.5 & 3 \\
\multirow{4}{*}{ Akita } & 10 & 15 & 4 & 3.9 & 4 \\
& 16 & 13 & 3.5 & 4.0 & 3.5 \\
& 5 & 18 & 4 & 3.5 & 4 \\
\hline \multirow{2}{*}{ Average values } & 10 & 14 & 3.5 & 4.0 & 3 \\
& 16 & 15 & 3.5 & 3.8 & 3 \\
& 10 & 19 & 4 & 4.1 & 4 \\
\hline
\end{tabular}

Table III. Statistics of persistence of planetary wave type oscillations in $f o F 2$ for stations from the Northern America and Japan based on the Morlet wavelet transform (absolute values). The average values for medians and the most frequent values are presented with a step of 0.5 .

\begin{tabular}{cccccc}
\hline \hline Station & Period (days) & Number of events & Median value & Mean value & Most frequent value \\
\hline Boulder & 5 & 45 & 4 & 4.4 & 3 \\
& 10 & 33 & 3.5 & 4.2 & 3 \\
Wallops Island & 16 & 32 & $3.5-4$ & 4.9 & 3 \\
& 5 & 49 & 4 & 4.3 & 3.5 \\
Wakkanai & 10 & 31 & 4 & 4.5 & $3.5-4$ \\
& 16 & 21 & 5 & 5.1 & 3 \\
Akita & 5 & 33 & 4 & 4.4 & 4 \\
& 10 & 34 & $3.5-4$ & 4.7 & 3.5 \\
& 16 & 52 & 4.5 & 4.1 & 3 \\
\hline \multirow{2}{*}{ Average values } & 10 & 38 & 4 & 4.5 & 4 \\
& 16 & 43 & 4 & 4.0 & 3.5 \\
\hline
\end{tabular}

Table IV. Statistics of persistence of planetary wave type oscillations in $f o F 2$ for stations from the Northern America and Japan based on the Morlet wavelet transform (relative values). The average values for medians and the most frequent values are presented with a step of 0.5.

\begin{tabular}{cccccc}
\hline \hline Station & Period (days) & Number of events & Median value & Mean value & Most frequent value \\
\hline \multirow{2}{*}{ Boulder } & 5 & 85 & 4 & 4.2 & 4 \\
& 10 & 59 & 3.5 & 4 & 3 \\
\hline
\end{tabular}


Table IV (continued).

\begin{tabular}{cccccc}
\hline \hline Station & Period (days) & Number of events & Median value & Mean value & Most frequent value \\
\hline \multirow{4}{*}{ Wallops Island } & 16 & 42 & 4 & 4.8 & 3 \\
& 5 & 89 & 4 & 4.4 & 4 \\
Wakkanai & 10 & 45 & 3.5 & 3.9 & 3.5 \\
& 16 & 35 & 3.5 & 4.2 & 3 \\
Akita & 5 & 98 & 4 & 4.4 & 4 \\
& 10 & 61 & 3.5 & 4.1 & 3.5 \\
& 16 & 106 & 3.5 & 3.8 & 3 \\
\hline \multirow{2}{*}{ Average values } & 5 & 55 & 4 & 4.5 & 4 \\
& 10 & 40 & $3.5-4$ & 4.1 & 3.5 \\
& 16 & 95 & 4 & 4 & 3 \\
\hline
\end{tabular}

Table V. Statistics of persistence of planetary wave type oscillations in $f o F 2$ over Europe based on the Morlet wavelet transform (relative values). The average values for medians and the most frequent values are presented with a step of 0.5 . After Laštovička et al. (2003a).

\begin{tabular}{cccccc}
\hline \hline Station & Period (days) & Number of events & Median value & Mean value & Most frequent value \\
\hline \multirow{2}{*}{ Juliusruh } & 5 & 45 & 4 & 4.5 & 4 \\
& 10 & 39 & 4 & 4.0 & $3+4$ \\
Slough & 16 & 35 & 3.5 & 3.8 & $3-3.5$ \\
& 5 & 37 & 4 & 4.3 & 4 \\
Průhonice & 10 & 29 & 3.5 & 3.6 & 3.5 \\
& 16 & 34 & 3.5 & 3.6 & $3-3.5$ \\
Rome & 10 & 49 & 4.5 & 4.7 & 4 \\
& 16 & 33 & 4.5 & 4.1 & 3 \\
& 5 & 31 & 4 & 3.8 & 3 \\
Average values & 10 & 33 & 3.5 & 3.0 & $3.5-4$ \\
& 16 & 25 & 3 & 3.5 & 3 \\
\hline
\end{tabular}

median values and the most frequent occurrences provide the typical persistence of 3.5 cycles. For the 16-day wave, medians point to a typical persistence of 3.5 cycles and the most frequent values reveal a typical persistence of just 3 cycles. Thus the persistence in terms of the number of cycles slightly decreases with increasing period. In terms of days the typical duration is 20 days for $T=5$ days, about 35 days for $T=10$ days and about 55-60 days for $T=16$ days. In other words, the duration of the wave events in terms of days is longer, not shorter for longer periods.

The median, mean and most frequent values are mostly very similar for all four stations and in tables II-IV. This means that American and Japanese stations reveal essentially the same statistical characteristics of persistence of the planetary wave type events in $f o F 2$, and that the «absolute» evaluation of the Paul wavelet transforms and the «absolute» and «relative» evaluation of 
Table VI. Statistics of persistence of planetary wave type oscillations in $f o F 2$ over Europe based on the Morlet wavelet transform (absolute values). The average values for medians and the most frequent values are presented with a step of 0.5. After Laštovička et al. (2003a).

\begin{tabular}{cccccc}
\hline \hline Station & Period (days) & Number of events & Median value & Mean value & Most frequent value \\
\hline Juliusruh & 5 & 55 & 4 & 4.8 & 4 \\
& 10 & 39 & 4.5 & 4.6 & 4.5 \\
Slough & 16 & $30+1^{*}$ & 4 & 4.8 & 3 \\
& 5 & 52 & 4.5 & 4.8 & $3-4$ \\
Průhonice & 10 & 38 & $4-4.5$ & 4.7 & 3.5 \\
& 16 & 33 & 4 & 4.3 & 3 \\
Rome & 10 & 42 & 4 & 4.6 & 4 \\
& 16 & 31 & 4.5 & 4.4 & 3 \\
& 5 & 55 & 4.5 & 4.7 & 4 \\
Average values & 10 & 52 & 4 & 4.9 & $3.5-4$ \\
& 16 & 38 & 4 & 4.6 & 4 \\
& 10 & 49 & $4-4.5$ & 4.8 & 4 \\
\hline
\end{tabular}

$1 *=\sim 220$ days long period of persistent occurrence in 1980 .

the Morlet wavelet transforms yield very similar statistical characteristics of the planetary wave type events.

The only substantial difference is in the number of events derived by different methods, as tables II-IV clearly demonstrate. However, it does not affect the statistical characteristics of the persistence. One source of differences between the «absolute» and «relative» evaluation is the existence of relatively long intervals of high but variable activity. Such an interval is evaluated as one event in «absolute» way, but may provide two or even three events in «relative» way due to that internal structure of the interval. The different number of identified events for the Morlet wavelet transform compared with the Paul wavelet transform is probably influenced by different mother wavelets.

Tables V and VI show statistical characteristics of the persistence of the planetary wave type events over Europe obtained with the Morlet wavelet transform. They are either identical with or very slightly larger than such values from the U.S.A. and Japan for all three periods of waves. This means that the statistical characteristics of planetary wave type event persistence are essen- tially the same in all three geographic regions and, thus, may be considered to be representative for middle latitudes of the Northern Hemisphere.

\section{Planetary wave type events in foF2 and the lower ionosphere over Europe}

As for the «vertical» comparison, we can compare the similarity of statistical characteristics of persistence of events, and of occurrence of individual planetary wave type events in $f_{o F} 2$ and the lower ionosphere in Europe using data and results of Laštovička et al. (2003a,b). The typical persistence of the 5-day wave in $f o F 2$ is four wave cycles (Laštovička et al., 2003b). This is slightly less than the typical persistence of 5 cycles for the 5-day wave in the lower ionosphere (Laštovička et al., 2003a). For the 10-day and 16-day waves, the typical persistence in $\mathrm{foF}_{2}$ and the lower ionosphere is identical. Thus the typical persistence of planetary wave type oscillations in the lower ionosphere and $f o F 2$ appears to be almost identical.

A quite different pattern is provided by comparison of individual events. The similarity of 
occurrence of individual events in $f_{o F} 2$ and the lower ionosphere is mostly poor, worse than expected from the results of some studies, which compared neutral wind oscillations in the MLT region with oscillations in $f o F 2$ or in the radio wave absorption in the lower ionosphere (e.g., Pancheva et al., 1994). The quasi-simultaneous occurrence of events in foF 2 and the lower ionosphere is rare. The reason might be that the absorption fluctuations are related to fluctuations in the prevailing wind (e.g., Pancheva et al., 1989), while those in $f o F 2$ seem to be related more to fluctuations in the tidal winds (e.g., Laštovička and Šauli, 1999). The planetary wave type fluctuations in the prevailing wind and the tidal wind differ to some extent. Why and to what degree these fluctuations differ should be examined. Another reason of the poor similarity might be the geomagnetic origin of a significant part of planetary wave type fluctuations in foF2 (Altadill and Apostolov, 2003), whereas the geomagnetic activity does not play a role in planetary wave type oscillations in the lower ionosphere (Pancheva et al., 1989). On the other hand, probably not all planetary wave type oscillations from the lower ionosphere have chance to be (indirectly) transported to heights of foF 2 .

\section{Longitudinal size of planetary wave events}

We analysed the planetary wave events for eight stations, which represented Europe, Japan, and the mid-latitude U.S.A. Similarity of occurrence (in the sense of simultaneous occurrence) of individual planetary wave type events observed in these three separated regions makes possible to estimate the longitudinal size of events or at least to put some constraints on that size. Altadill and Apostolov (2003) used another approach and found a typical longitudinal size of the planetary wave type events in foF 2 as follows:

$$
\begin{aligned}
& T=5-6 \text { days, } 80^{\circ} \\
& T=10 \text { days, } 100^{\circ} \\
& T=16 \text { days, } 180^{\circ}
\end{aligned}
$$

with individual events covering up to the whole globe.

Our analysis reveals that there is almost no simultaneous occurrence of the $T=5$ days events observed in Europe, Japan and US, even though statistical characteristics of their persistence are closely similar. Table VII shows an example of such a $T=5$ days event. The event first occurs in US (Wallops), immediately after the end of the event in US it appears in Europe, and a few days after the end of the event in Europe it appears in Japan, which looks like an eastward propagation of an event of limited longitudinal size. However, if we consider the longitudinal difference between the regions (stations) and the typical longitudinal size of $80^{\circ}$ found by Altadill and Apostolov (2003), the observed absence of simultaneous occurrence of wave events coincides with the typical longitu-

Table VII. A $T=5$ days event and a $T=10$ days event, both observed in the second half of 1986, Morlet wavelet transform. Too short means shorter than 3.0 cycles; short bursts means only short, separated bursts occur.

\begin{tabular}{ccccccc}
\hline \hline $\begin{array}{c}\text { Period } \\
\text { station }\end{array}$ & Days & $\begin{array}{c}5 \text { days } \\
\text { Cycles }\end{array}$ & Duration & Days & $\begin{array}{c}10 \text { days } \\
\text { Cycles }\end{array}$ & Duration \\
\hline Juliusruh & $300-316$ & 3.2 & 16 days & $245-279$ & 3.4 & 34 days \\
Slough & Too & Short & & $240-283$ & 4.3 & 43 days \\
Průhonice & $300-316$ & 3.2 & 16 days & $240-281$ & 4.1 & 41 days \\
Roma & Short & Weak & & Too & Short & \\
Boulder & Too & Short & & No & Effect & \\
Wallops & $266-299$ & 6.6 & 33 days & Short & Bursts & \\
Wakkanai & $320-336$ & 3.2 & 16 days & No & Rffect & \\
Akita & $320-335$ & 3.0 & 15 days & No & Effect & \\
\hline
\end{tabular}


dinal size of such events established by Altadill and Apostolov (2003).

We analysed several $T=7$ days events. Such events observed at European, Japanese and US stations showed again almost no simultaneous occurrence of individual events. The $T=10$ days events also mostly displayed absence of simultaneous occurrence, as the event shown in table VII, in correspondence to their typical longitudinal size of $100^{\circ}$ as found by Altadill and Apostolov (2003).

On the other hand, a few $T=16$ days events, which were studied, revealed much often simultaneous or partly simultaneous occurrence of wave events between European, US and Japanese stations. One such event happened in 1984 with beginning around day 60 and persistence of four wave cycles in Europe (at all four stations), and beginning slightly later and persisting again about four wave cycles for Wakkanai, Boulder and Wallops Island (at southernmost Akita persistence shorter than three wave cycles). This is consistent with their much larger typical longitudinal size found by Altadill and Apostolov (2003).

\section{Discussion}

We investigate the persistence of planetary wave type oscillations over Europe among others in order to clarify possible predictability of such oscillations with applications to the predictions of the radio wave propagation conditions. For the sake of predictions, not only the typical values of the persistence of planetary wave type events, but also the spectral distribution of persistence of individual events (= event duration) is required. An example of the spectral distribution of event duration is shown in fig. 3, which presents for each region one station. The spectral distribution of event duration is too broad to allow a reasonable prediction of event duration from foF 2 measurements themselves. Moreover, figs. 1 and 2 display many events of duration shorter than three cycles, which is the lower limit of studied events. Thus the predictability of the planetary wave type oscillations in $f_{o} F 2$ from the measurements of fof 2 itself seems to be very questionable. We

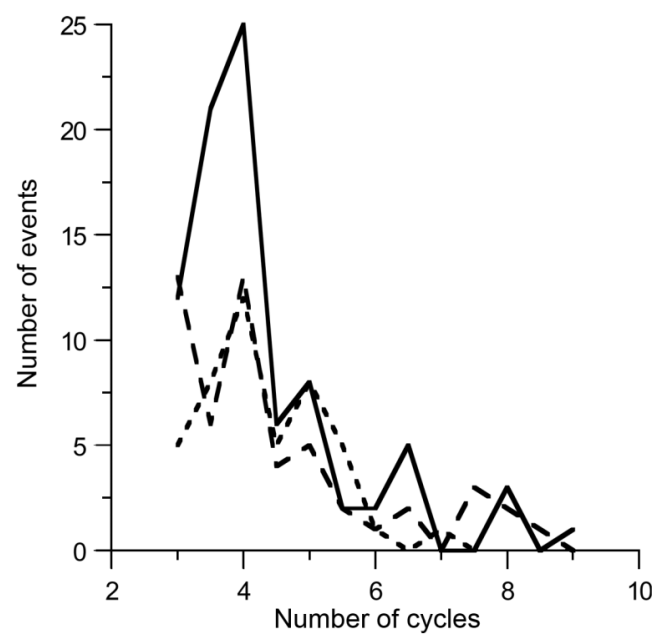

Fig. 3. Spectra of event duration (number of wave cycles) for the 5-day oscillations, Morlet wavelet transform for Boulder («absolute» evaluation, solid curve), Akita («relative» evaluation, long-dash curve), and Juliusruh («absolute» evaluation, short-dash curve).

have to look for other predictors, which is complicated due to different physical origin of different planetary wave type events. Unfortunately, we will probably be unable to separate predictable planetary wave type events, which mean that they remain a part of the prediction noise. Improvement of the quality of radio wave propagation predictions in such a way seems to be rather impossible, or at least very difficult.

Some planetary wave events have sharp onset and end. Then the determination of planetary wave persistence depends on the time/period resolution of the used wavelet. Shape of selected wavelet influences how the wavelet captures/detects each wave-like event. However, the onset and end of most events is not so sharp and the accuracy of the determination of onset duration in such cases may be up to a few days, particularly for the «relative» evaluation. Therefore we can consider the accuracy of determination of the typical persistence of planetary wave events to be rather around half a cycle for 5-day waves, and better for longer periods in terms of wave cycles. 


\section{Conclusions}

The basic characteristics of the $F 2$ region, the critical frequency foF 2 , were analyzed for two US stations, Boulder and Wallops Island, two Japanese stations, Akita and Wakkanai, and the results were compared with the results of Laštovička et al. (2003b) for four European stations Juliusruh, Slough, Pruhonice and Rome, and Laštovička et al. (2003a) for the lower ionosphere above Central Europe. All data were analyzed over the period 1979-1989 (one solar cycle). Noontime average values (10-14 UT) of foF 2 were used. The persistence of planetary wave type oscillations at periods near 5, 10 and 16 days and sometimes 7 days was studied with the use of the Paul, Morlet and Meyer wavelet transforms. Only events of duration of three cycles and more were considered. The main results are as follows:

1) There is a large temporal and partly spectral variability of planetary wave type activity. The migration of periods of planetary wave activity is also well visible. The large temporal variability of the planetary wave activity results in a limited persistence of the individual planetary wave type events.

2) For the 5-day wave, a typical persistence of well-developed wave events in $f o F 2$ is 4 wave cycles. For the 10-day wave, it is rather 3.5 wave cycles. For 16 days, the most frequent values provide typical persistence no more than 3 wave cycles.

3) In terms of the number of wave cycles in the planetary wave type events, the persistence decreases towards longer periods. However, the persistence of wave events in terms of days increases towards longer periods.

4) The planetary wave type wave persistence characteristics for Europe are either identical with or very slightly larger than corresponding values from the U.S.A. and Japan.

5) The spectrum of event duration is very broad. The character of the spectrum does not allow us to predict the duration of an event when we observe its beginning or, say, first 2-3 wave cycles.

6) The longitudinal size of the planetary wave type events increases with increasing period, making the 5-day and 10-day period events in Europe, America and Japan essentially dissimilar, and the 16-day oscillations much more similar among the three regions.

7) While the typical persistence of planetary wave type oscillations in $f_{o F} 2$ and the lower ionosphere over Europe is similar, the correspondence of occurrence of individual events is rather poor.

\section{Acknowledgements}

This work has been supported by the Academy of Sciences of the Czech Republic under project AVOZ30420517.

\section{REFERENCES}

Altadill, D. and E.M. Apostolov (2001): Vertical propagating signatures of wave type oscillations (2 and 6.5 days) in the ionosphere obtained from electron density profiles, J. Atmos. Solar-Terr. Phys., 63, 823-834.

Altadill, D. and E.M. Apostolov (2003): Time and scale size of planetary wave signatures in the ionospheric $F$ region. Role of the geomagnetic activity and mesosphere/lower thermosphere winds, J. Geophys. Res., 108 (A11), 1403, doi: 10.129/2003JA010015.

Altadill, D., E.M. Apostolov, J. BoŠKA, J. LAŠtovičKa and P. ŠAULI (2004): Planetary and gravity wave signatures in the $F$-region ionosphere with impact on radio propagation predictions and variability, Ann. Geophysics, 47 (suppl. to n. 2/3), 1109-1119.

BUREŠOVÁ, D. (1997): Results of foF2 data testing with the UNDIV program, Studia Geophys. Geod., 41, 82-87.

CAVALIERI, D.J. (1976): Travelling planetary-scale waves in the E-region ionosphere, J. Atmos. Terr. Phys., 38, 965974.

DAUBECHIES, I. (1994): Ten lectures on wavelets, CBMS, SIAM, 61, 117-119.

Forbes, J.M. and X. ZHANG (1997): Quasi 2-day oscillation of the ionosphere: a statistical study, J. Atmos. SolarTerr. Phys., 59, 1025-1034.

Forbes, J.M., S. PALO and X. ZHANG (2000): Variability of the ionosphere, J. Atmos. Solar-Terr. Phys., 62, 685-693.

Haldoupis, C., D. Pancheva and N.J. Mitchell (2004): A study of tidal and planetary wave periodicities present in mid-latitude sporadic E layers, J. Geophys. Res., 109, A02302, doi: 10.1029/2003JA010253.

Kazimirovsky, E.S., M. Herraiz and B.A. DE LA Morena (2003): Effects on the ionosphere due to phenomena occurring below it, Surv. Geophys., 24, 139-184.

LAŠTOVIČKA, J. (2006): Forcing of the ionosphere by waves from below, J. Atmos. Solar-Terr. Phys., 68, 479-497.

LAŠTOVIČKA, J. and P. ŠAULI (1999): Are planetary wave type oscillations in the $F 2$ region caused by planetary wave modulation of upward propagating tides?, $A d v$. Space Res., 24, 1473-1476.

LAŠTOVIČKA, J., V. FIŠER and D. PANCHEVA (1994): Long- 
term trends in planetary wave activity (2-15 days) at 80-100 km inferred from radio wave absorption, J. Atmos. Terr. Phys., 56, 893-899.

LAŠTOVIČKA, J., P. KRIŽAn and D. Novotná (2003a): Persistence of planetary waves in the lower ionosphere, Studia Geophys. Geod., 47, 161-172.

LAŠTOVIČKA, J., P. KRIŽAN, P. ŠAUli and D. Novotná (2003b): Persistence of the planetary wave type oscillations in foF 2 over Europe, Ann. Geophysicae, 21, 1543-1552.

Mallat, S. (1998): A Wavelet Tour of Signal Processing (Academic Press, San Diego), pp. 577.

PAnCheVA, D., E. Apostolov, J. LAŠTovičKa and J. BošKA (1989): Long-period fluctuations of meteorological origin observed in the lower ionosphere, J. Atmos. Terr. Phys., 51, 381-388.
Pancheva, D., L.F. Alberca and B.A. DE LA Morena (1994): Simultaneous observation of the quasi-two day variations in the lower and upper ionosphere, J. Atmos. Terr. Phys., 56, 43-50.

Rishbeth, H. and M. Mendillo (2001): Patterns of ionospheric variability, J. Atmos. Solar-Terr. Phys., 63, 1661-1680.

Torrence, C. and G.P. Compo (1998): A practical guide to wavelet analysis, Bull. Amer. Met. Soc., 79 (1), 61-78.

VIDAKOvic, B. (1999): Statistical Modelling by Wavelets (A Wiley-Interscience Publication, John Wiley \& Sons), pp. 408.

(received October 14, 2005; accepted May 17, 2006) 